\title{
An Efficient Cross-tier Interference Mitigation Technique in LTE Femtocell Environment
}

\author{
A. Habib, Waseem Ullah Khan, Tahir Ullah \\ Department of Computer Systems Engineering \\ University of Engineering \& Technology, Peshawar
}

\begin{abstract}
Demand for high speed, innovative and up- to- date technologies is always on the rise. In terms of capacity, cellular networks are near to their physical limit. But the expansion of data services still increasing exponentially. The shift from legacy computers to smartphones made things even more complex by introducing hungry applications demanding high data rates such as Web browsing, email, video sharing, social networking, online interactive games, and locationbased services, etc. All such services and obviously future services are bandwidth hungry and can't be bounded to certain areas only. To provide such services for outdoor users is not a significant issue but it becomes a challenging task for service providers to offer such services for indoor users. The strength of the signal is deteriorated quite significantly due to penetration-losses from different physical barriers including walls and floors. In order to meet the demand of providing good quality signal strength indoor, one of the most promising solutions provided by the 3rd Generation Partnership Project (3GPP) for improving capacity, speed and signal strength is the deployment of femtocell for future wireless networks. Femtocell has a small cell based architecture. It is a plug and play, fully featured, low-powered cellular base station residing indoors where usually a poor signals are received from outdoor base stations. However, their deployment brings many challenges. Interference management is one of them in case of their uncoordinated deployment in the coverage range of macro base stations. A femtocell based method with controlled power is designed and implemented to mitigate cross-tier downlink interference between the macro base station and femtocell users. In this paper, the feasible transmission power of femtocell is determined with respect to user locations in different scenarios and to achieve targeted signal-to-interference-plus-noise-ratio (SINR) values. Performance analysis in various scenarios of the proposed method is presented. The results of proposed scheme can improve system performance in terms of received signal strength.
\end{abstract}

\section{General Terms}

Femtocell, Cross-tier interference, Indoor coverage.

Keywords

3GPP, SINR, Downlink, Data rate.

\section{INTRODUCTION}

In the beginning, mobile communication mostly dominated by voice traffic. At that stage the requirements for mobile data is minimal, but after 2012 the rapid increase in demand of mobile data has compelled wireless service providers and equipment vendors to explore new ideas and concepts to fulfill the clients demand by providing high data rates particularly for indoor users. Ericsson observed mobile data generated across the globe in-between February 2012 to August 2018 [1]. During this period data traffic increases almost $71 \%$ annually. This tendency is set to continue across the region fueled by a mobile broadband strong comeback in the recent years.

Due to the mobility of modern society, the need for satisfactory coverage at dead spots or weak-signal areas have become prominent issues in the today's cellular systems. Most of contemporary services offered by cellular operators are $3 \mathrm{G}$ and 4G. Data traffic in dead spot areas where high data services cannot be accommodated efficiently due to poor signal strength. Furthermore, the expansion of data services is looking inevitable and traffic volumes are rapidly increasing day by day. Figure 2 shows forecast of mobile data and their expansion of almost $55 \%$ annually in the period from 2020 to 2030 [2].

Cellular network planners were initially intended to design wide area network coverage and successfully deployed systems for robust outdoor coverage. However, to sustain steady indoor coverage with good quality of strength is a challenging task for network operators. For this, much more effort and investment are required because the signal is attenuated by a different number of factors including windows, roofs, and walls. As an outcome, the same application running on the same User Equipment (UE) utilizes much more resources of the network than if they were outdoors. So, the effective capacity of the network decreases as indoor users increases.

One promising concept to fulfill these requirements and challenges is the deployment of femtocell having services range in-between 10-30 meters at homes and in small buildings. Femtocell enables a remarkable increase in network density and spatial-frequency reuse.

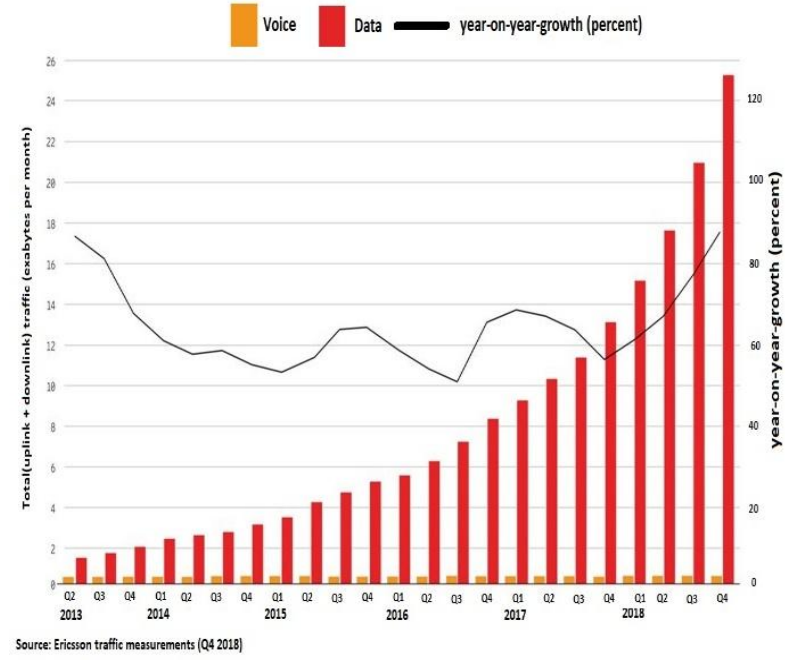

Figure 1: Mobile traffic (data and voice) generated across the World "2012 to 2018" [1] 


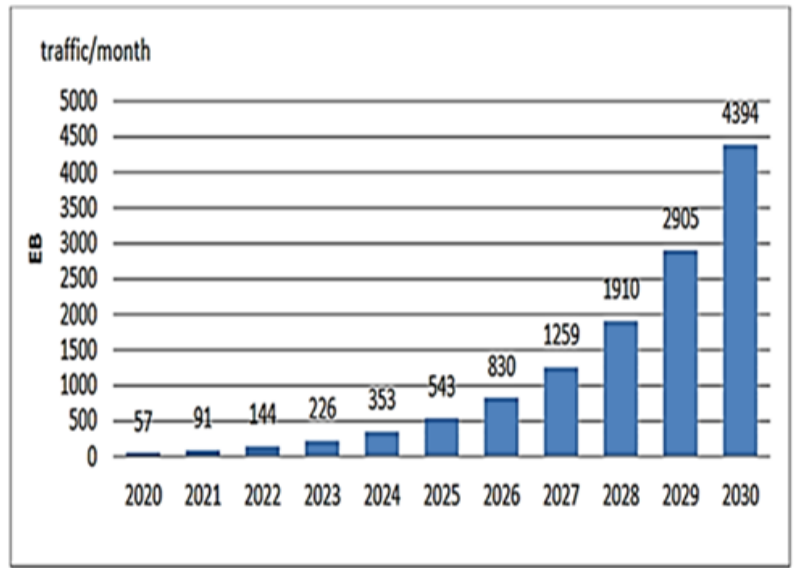

Figure 2: Data forecast worldwide in the period from 2020 to 2030 [2]

\section{FEMTOCELL TECHNOLOGY}

Femtocell is a low power cellular base station, which offers improved indoor coverage with better performance using licensed spectrum and stable internet connection to connect to the operator's core network. It is a plug and play device placed indoors having a poor quality of signals receiving from base station due to propagation path loss of the outer walls as well as inner-floor. The aggregation of these losses can reach a considerable level, thus making indoor coverage very challenging. So relying on coverage provided by outdoor base station located few kilometers away is not an attractive concept to effectively provide high-quality indoor coverage. Thus femtocell is a promising solution with friendly indoor propagation environment with high SINR to provide improved performance.

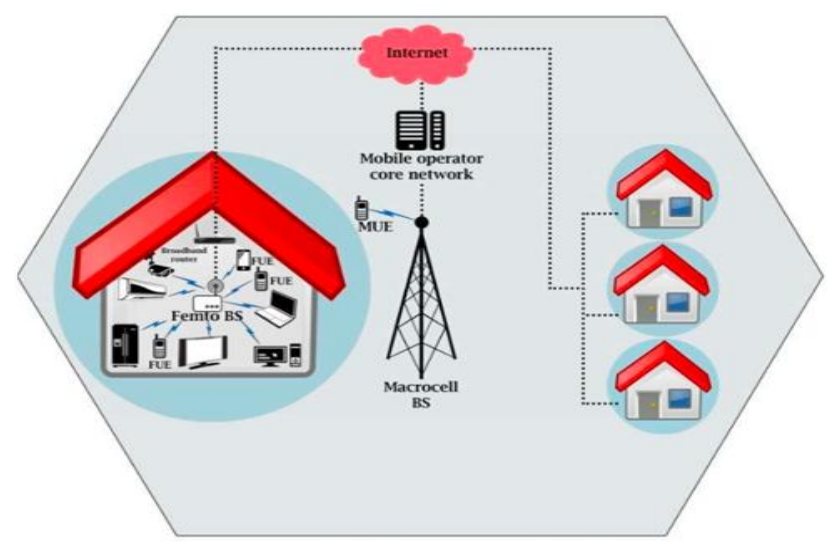

Figure 3: Typical Indoor Femtocell Architecture in LTE [3]

Femtocell provides high multimedia and data services at a reasonable price target. It radiates low power $(<10 \mathrm{~mW})$ and can support typically 5 to 10 users. In case of a residential scenario, traffic generated indoor are routed to homes ISP connection encouraged by cellular operators to initiate new services, applications and discover new ideas for revenue generations. Figure 3 shows a typical indoor OFDMA femtocell architecture in LTE.

\subsection{Access Mode of Femtocell}

Femtocell operates in three different operating modes namely, Open Access Mode (OAM), Closed Access Mode (CAM) and Hybrid Access Mode (HAM).

\subsubsection{OAM}

In open access mode, all users (femto user and macro user) are treated equally. Any user comes into femto coverage area enjoy full service without any restrictions. It is deployed by network operators in the poor coverage area. This is like the hotspot scenario to extend services in dense public or community areas just like coffee shops, universities, airports, and stadiums, etc.

\subsubsection{CAM}

In this mode, only subscriber or registered user belonging to Closed Subscriber Group (CSG) are authorized to connect to femtocell. Users other than CSG are not allowed to connect.

\subsubsection{HAM}

This mode is just like CSG mode but supports only limited access for the unsubscribing user. It is somewhat in-between closed and open access mode.

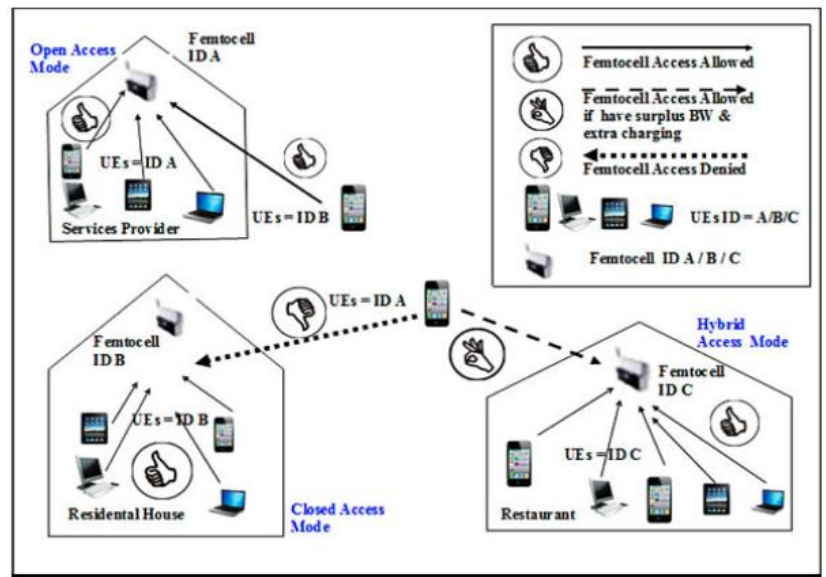

Figure 4: Different Access Mode Permission in Femto Environment [4]

\subsection{Technical Issues Regarding Femtocell Deployment}

Although femtocell technology is promising technology but there are some technical challenges related to this technology.

\subsubsection{Frequent Handover.}

Femtocell with small coverage range is deployed inside the wireless network. In open access mode, handover issues are generated. Normally users are connected to femtocell inside the building when user move outside the coverage area of FAP it will be connected to MBS. Similarly, if a user from outside come in the range of femtocell handover from MBS to femto are made. Due to user mobility, an efficient handover algorithms are required. Such a frequent handover creates unnecessary load on the network.

\subsubsection{Channel Allocation}

Efficient allocation of the channel is very important for successful deployment of a femtocell. If separate channels are allocated for Marco and Femto then the efficiency of the spectrum will be compromised. In co-channel deployment, a better spectral efficiency will be achieved compared to dedicated channel, but interference will become a prominent issue.

\subsubsection{Stable Internet Connection}

High internet services such as DSL or Optic Fiber are used to connect Femtocell to operator's core network. So, for successful data transfer a stable internet connection is 
required. Otherwise it can cause problem in data transfer and voice calls.

\subsubsection{Power Control}

The efficient control power mechanism are required in order to minimize the interference issues. In case of co-channel deployment, a high power femtocell transmission can affect any user connected to MBS because femto signal acts as an interference signal to that user.

\subsubsection{Interference Management}

The interference management is the major issue in effective deployment of femtocell. It operates on licensed spectrum due to which a careful inference management techniques are required keeping the spectral efficiency in view.

\section{LITERATURE RIVIEW}

A notable amount of literature related to femtocell interference mitigation both in Co-tier and Cross-tier are available. In Co-tier interference occurs between the same tiers of network elements. For example, Such as interference among neighboring femtocells. Cross-tier interference occurs between dissimilar tiers of network elements. Such as interference between the macrocell tier and those of the femtocell tier and vice versa.

In [5] Femto-cell Power Control Scheme (FPCS) is presented. This scheme adopted Analytical approach to femto base stations transmit power.

In [6] Fair Downlink Power Control Algorithm for Femtocell Networks is presented. It used both split and shared spectrum. It based on assumptions provided equal signal strength to most of users.

In [7] Power based control schemes PC-1 and PC-2 are presented. They estimated the SINR values of both macro and femto user. PC-1 used fixed SINR threshold while PC-2 used dynamic SINR determined by PC-2 algorithm. These methods is for uplink interference mitigations.

\section{NETWORK MODEL AND PROBLEM DESCRIPTION}

A two-tier heterogeneous model is considered comprising of femtocell embedded with single macrocell as shown in Figure 5.

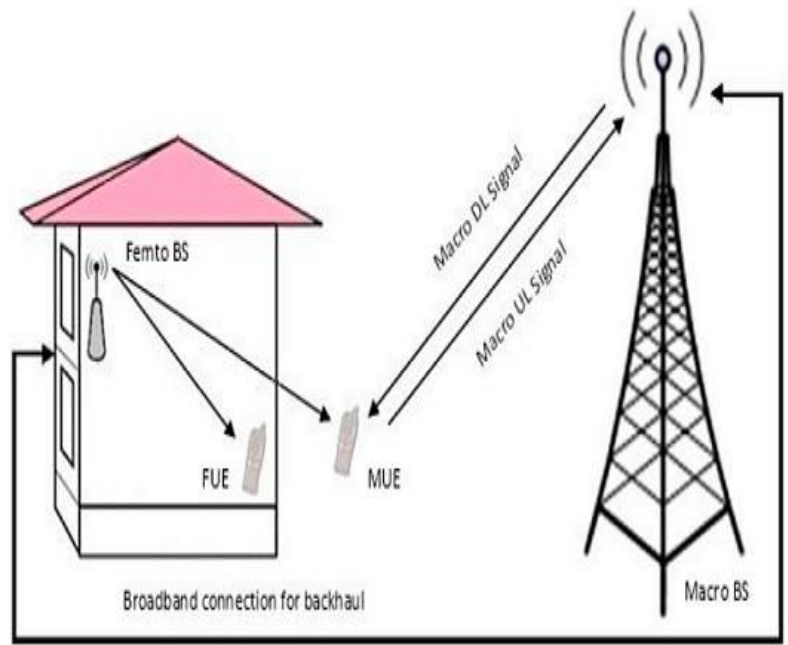

Figure 5: The Deployment of Femtocell inside LTE macrocell [8]
Both MBS and FBS are considered using the same Orthogonal Frequency Division Multiple Access (OFDMA) and Single Carrier Frequency Division Multiple Access (SCFDMA) technologies on downlink and uplink respectively operated on the same licensed spectrum. It is assumed that MBSs provide complete coverage but there are some loopholes where coverage is very poor such as in dense public areas where for the purpose of improving network coverage, traffic offloading from macrocell and increasing capacity femtocell is deployed as a hotspot. With pre-planning regarding infrastructure, interference management in femtocell deployment becomes very important. To make analysis convenient, some assumptions are made:

Assumption 1: This method is purely presented for crosstier interference (It is resulting from the occurrence of interference due to dissimilar tiers. E.g. interference between macrocell tier and femtocell tier vice versa).

Assumption 2: Access method of Femtocell is OAM.

Assumption 3: Any subscriber or user can be served by at least one Base Station (BS).

\subsection{Dynamically Assigned Power Interference Reduction Algorithm (DAPIR)}

In this paper, a novel method is proposed for better performance of femtocell in terms of interference management. The main idea behind this scheme is to attain controlled femtocell interference based on channel quality indicator (CQI) report generated by MBS. This report already contains interference due to nearby femtocell. Thus, depending on this value the femtocell adjust it's transmit power so that SINR seen by the affected macro-cell user (MUE) due to FBS remains in tolerable range. This report is generated after every Transmission Time Interval (TTI) expired and shared among femtocells located near macrocell. The value of TTI is carefully selected. The femtocell observes CQI report generated by the MBS having detail about the affected macro user in its vicinity.

When femtocell is plugged in, it collects information about its position, potential interferer with their transmission power and penetration loss associated with its serving building. The whole process is repeated every time when it is booted.

The overall operation of the proposed algorithm is controlled by two key decision making parameters by FBS.

1) If Noise level is greater than the threshold (threshold Noise level) for detecting any active user and disturbing (interference)

2) The strength of the signal, pilot signal, and interfering signal. Power of the received signal to potential interferer (Prec).

Noise level can be calculated as:

$$
\text { NOISE_UPLk }=\sum_{u e=1}^{N_{u e}}\left(P_{u e} P \operatorname{LosS}\left(d_{u e, k}\right)\right)
$$

$N_{u e}$ is the total number of active subscriber within Macrocell. Where $P_{u e}$ represents UE transmitting power, $\operatorname{PLoss}\left(d_{u e, k}\right)$ is path loss between UE and FBS.

FBS estimates and compare key decision-making parameters (NOISE_UPLk, Prec) with threshold and calibrates its power accordingly. The optimization algorithm is summarized below 
1) Suppose there is an active user in the coverage area of femtocell NOISE_UPLk $\geq$ NOISE_TH $\& \&$ Pmacro $\geq$ Pfemto if both these parameters exceeded the threshold value then femtocell decreased its power to minimized level in order to minimize the interference level.

$$
\boldsymbol{P}_{\text {femto }}=\boldsymbol{P}_{\text {rec, }, \mathrm{K}}^{M B S}+\boldsymbol{P}_{L}\left(\boldsymbol{R}_{\text {min }}\right) \cdot \boldsymbol{P}_{\text {femto }, \text { min }}
$$

2) If NOISE_UPLk $\geq$ NOISE_TH \&\& Pmacro $\leq$ Pfemto then, in this case, femtocell has to increase its power to provide QoS for the edge user and at the same time handover takes place based on assumption 3 as stated earlier.

$$
P_{\text {femto }}=P_{\text {rec }, k}^{M B S}+P_{L}\left(R_{\text {max }}\right) \cdot P_{\text {femto }, \text { max }}
$$

3) If both above parameters failed then continue with normal power.

$$
P_{f e m t o, i n i}=P_{r e c, k}^{M B S}+P_{L}\left(R_{\text {ini }}\right) \cdot P_{f e m t o}
$$

In DAPIR, Where $P_{\text {rec, } k}^{M B S}$ is the MBS transmitting power received by femtocell. $P_{L}(R)$ is the pathloss from femtocell to user $\mathrm{UE}$ at radius $\mathrm{R}$. Therefore, the authors define three levels of coverage radius i.e. Rini, Rmin and Rmax and used it to contain the coverage according to the situation.

\section{PATH LOSS MODELS}

To estimate the overall capacity of the network path loss models play an important factor, it is a good instrument and widely used for network capacity estimation. It depends on various environmental factors. Various path loss models are present in literature both for urban and suburban areas. But so far the following model between UE and FBS and MBS are used.

$$
\begin{aligned}
& P L_{N L O S}=128.1+37.6 * \log 10\left(r_{u, M B S}\right)+\text { Noise }- \\
& \text { Noise floor }
\end{aligned}
$$

Where NLOS denotes that there is no visual line of sight between transmitter and receiver. $r_{u, M B S}$ is the distance between MBS and UE.

$$
\begin{aligned}
& P L_{N L O s}=128.1+37.6 * \log 10\left(l_{u, k}\right)+\text { Noise }- \\
& \text { Noise floor }
\end{aligned}
$$

$\left(l_{u, k}\right)$ is the distance between $\mathrm{UE}$ and $\mathrm{FBS}_{\mathrm{k}}$. . This equation is used for femto user inside the house.

$$
\begin{aligned}
& P L_{N L O S}=128.1+37.6 * \log 10\left(l_{u, k}\right)+\text { Noise }- \\
& \text { Noise floor }+\mathrm{L}_{\mathrm{wl}}
\end{aligned}
$$

$L_{w l}$ is the wall penetration loss inside the building. This equation is used for a macro user outside the house containing femtocell but receiving a strong signal from HeNB.

With the help of these path loss models the Threshold value Noise_Th has been derived. The simulation will show that DAPIR algorithm perform well if any of the path models are used.

\section{SIMULATION PARAMETERS}

Table 1 summarizes the different assumptions, threshold values and general parameters used in these simulations. Based on these parameters various scenarios are evaluated. Femtocell in this scenario is placed in the small apartment $5 \mathrm{X}$ $5 \mathrm{~m}$ within the coverage range of Macrocell. Wall penetration loss is assumed to be $20 \mathrm{~dB}$ [8]. The performance of

\begin{tabular}{|c|c|c|}
\hline Parameters & Symbol & Values \\
\hline $\begin{array}{c}\text { Downlink Operating } \\
\text { frequency }\end{array}$ & $f$ & $20 \mathrm{GHz}$ \\
\hline $\begin{array}{l}\text { Distance between } \\
\text { eNodeB and HeNB }\end{array}$ & $d$ & $700 \mathrm{~m}$ \\
\hline Initial femtocell radius & $R_{\text {ini }}$ & $10 \mathrm{~m}$ \\
\hline $\begin{array}{l}\text { Minimum femtocell } \\
\text { radius }\end{array}$ & $R_{\min }$ & $5 \mathrm{~m}$ \\
\hline $\begin{array}{l}\text { Maximus femtocell } \\
\text { radius }\end{array}$ & $R_{\max }$ & $15 \mathrm{~m}$ \\
\hline Transmit Power of MBS & $P_{t}^{M B S}$ & $46 \mathrm{dBm}$ \\
\hline $\begin{array}{c}\text { Initial Transmit Power of } \\
\text { FBS }\end{array}$ & $P_{\text {femto }}$ & $15 \mathrm{dBm}$ \\
\hline $\begin{array}{l}\text { Minimum Transmit } \\
\text { Power of FBS }\end{array}$ & $P_{\text {femto,min }}$ & $5 \mathrm{dBm}$ \\
\hline $\begin{array}{l}\text { Maximum Transmit } \\
\text { Power of FBS }\end{array}$ & $P_{\text {femto, } \max }$ & $23 \mathrm{dBm}$ \\
\hline Site Layout & eNodeB & 1 \\
\hline $\begin{array}{l}\text { Maximum Transmit } \\
\text { Power of UEs }\end{array}$ & $P_{u e}$ & $23 \mathrm{dBm}$ \\
\hline Total number of FBS & $N$ & 1 \\
\hline Wall Penetration Loss & $L_{w l}$ & $20 \mathrm{dBm}$ \\
\hline User velocity & & Zero \\
\hline Cell Radius & $R_{\text {Macro }}$ & $1400 \mathrm{~m}$ \\
\hline Traffic environment & & Loaded \\
\hline Mobility Model & & $\begin{array}{c}\text { Random } \\
\text { Point }\end{array}$ \\
\hline
\end{tabular}
Dynamically Assigned Power Interference Reduction
(DAPIR) algorithms is evaluated using three different scenarios. Such as user located at the cell edge, conflicting zone and macro user deep inside the coverage area of femtocell. The performance of the DAPIR is compared with a fixed power, No Femtocell (No HENB) and illustrated the results.

Table 1: Simulation Parameters

Table 2: Different type of Scenarios used in Simulations

\begin{tabular}{|c|c|}
\hline Scenarios & UE locations \\
\hline Scenario 1 & UE at cell-edge of Macrocell \\
\hline Scenario 2 & $\begin{array}{r}\text { UE inside femtocell coverage area } \\
\text { but outside house }\end{array}$ \\
\hline Scenario 3 & UE located in conflicting zone \\
between MBS and FBS
\end{tabular}




\subsection{Sensitivity Analysis}

In this research, sensitivity analysis is performed to find the optimum values of different parameters. Considering the complexity of the system the sole purpose of sensitivity analysis is to find the values to increase the performance and capacity of the system. After studied various literature and running simulation about 50 number time the optimum value for NOISE_TH is selected as $-2 \mathrm{dBm}$. The time required to generate a $C Q I$ report by MBS is very important. Based on this report DPIR takes its decision. If $C Q I$ report generation time is longer than handover event than this produce bad impact on our algorithm and result might be erroneous. After study various literature the minimum time to complete handover must be less than $100 \mathrm{~ms}$ otherwise link can be failed [9]. Based on this handover event time transmission time interval (TTI) is selected to be $30 \mathrm{~ms}$. So MBS generate CQI report after every $30 \mathrm{~ms}$ and shared this report among all FBSs located in its proximity.

\section{RESULT AND ANALYSIS}

The scheduling of all UEs within its cells is done using Proportional Fair (PF) scheduler in this simulation. This scheduler considers both fairness and throughput. The PF scheduler distributes resources among users by taking into consideration their channel quality and their past average channel quality [10].

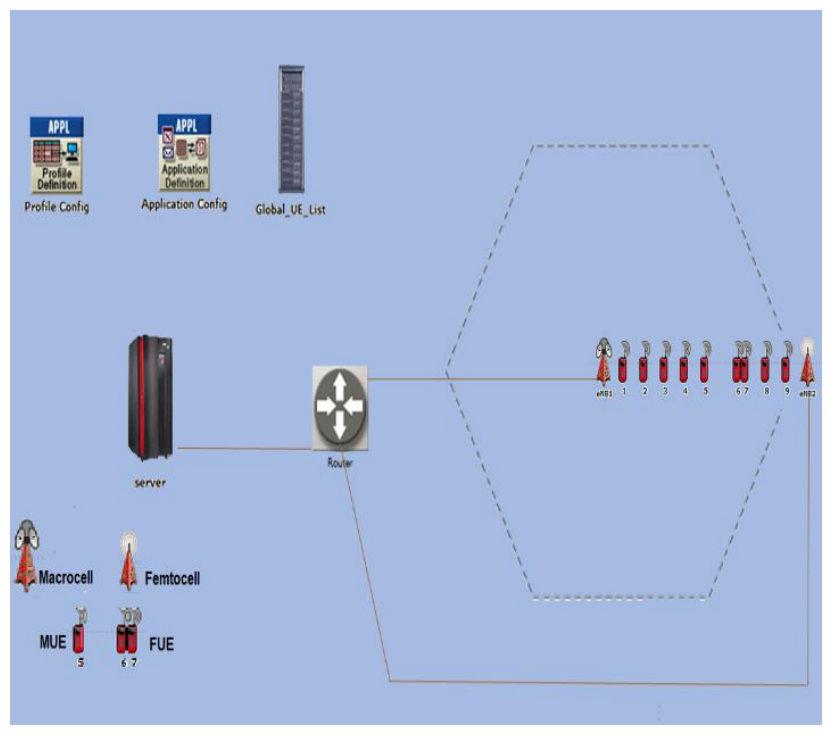

Figure 6: Simulation Scenario of a Reference Model

\subsection{Scenario 1}

In scenario 1, suppose the macro user MUE is located near the edge of the macrocell. It received both the signals (each from MBS as well as FBS). After applying DAPIR algorithm results are illustrated as shown in Figure 7 below which clearly outperformed other two in scenario 1.

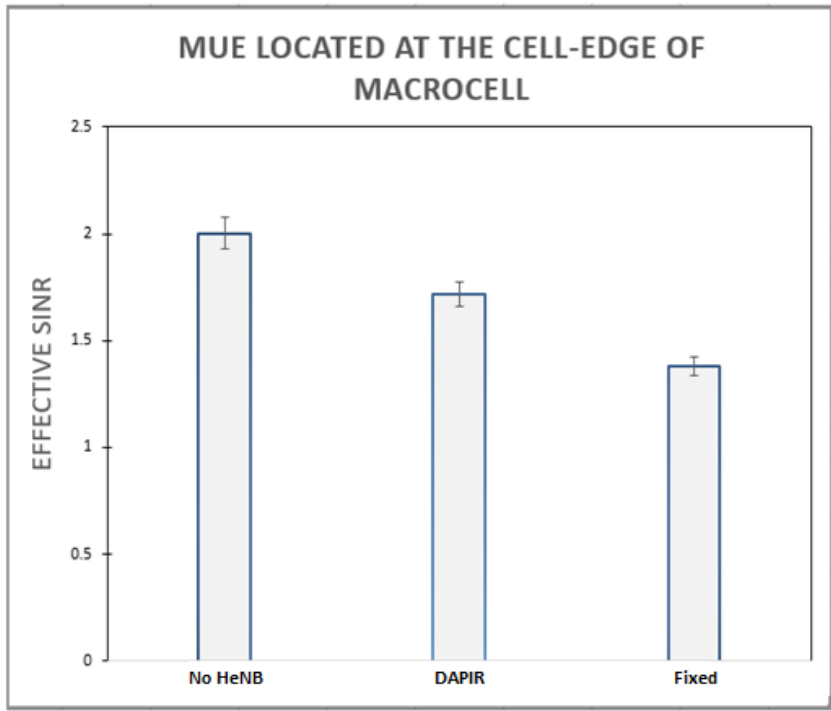

Figure 7: SINR Results of MUE located at Edge of Macrocell

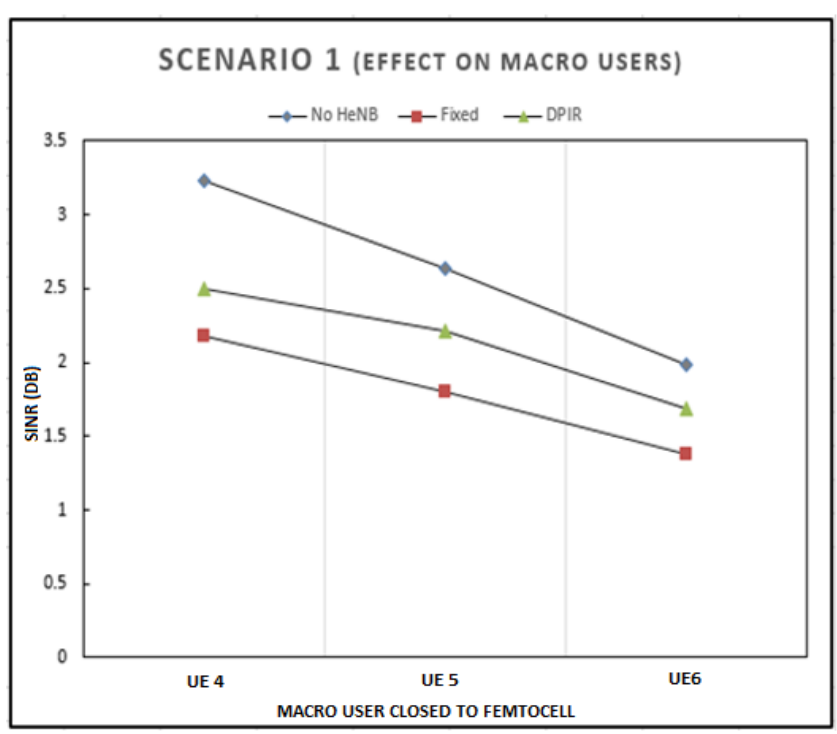

Figure 8: The effect of DAPIR on other users in macrocell based on Scenario 1

\subsection{Scenario 2}

In scenario 2, suppose macro user MUE penetrated deep inside the coverage area of femtocell. The noise level is too high to communicate with MBS. After applying DAPIR algorithm a significant improvement is observed. 


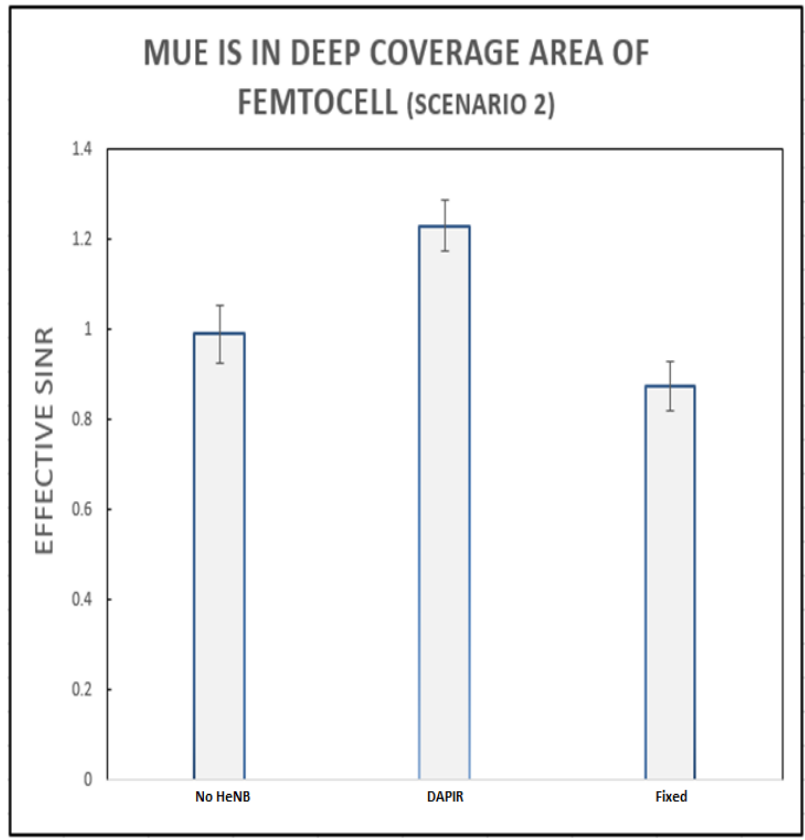

Figure 9: SINR Result of MUE is in Coverage Area of Femtocell

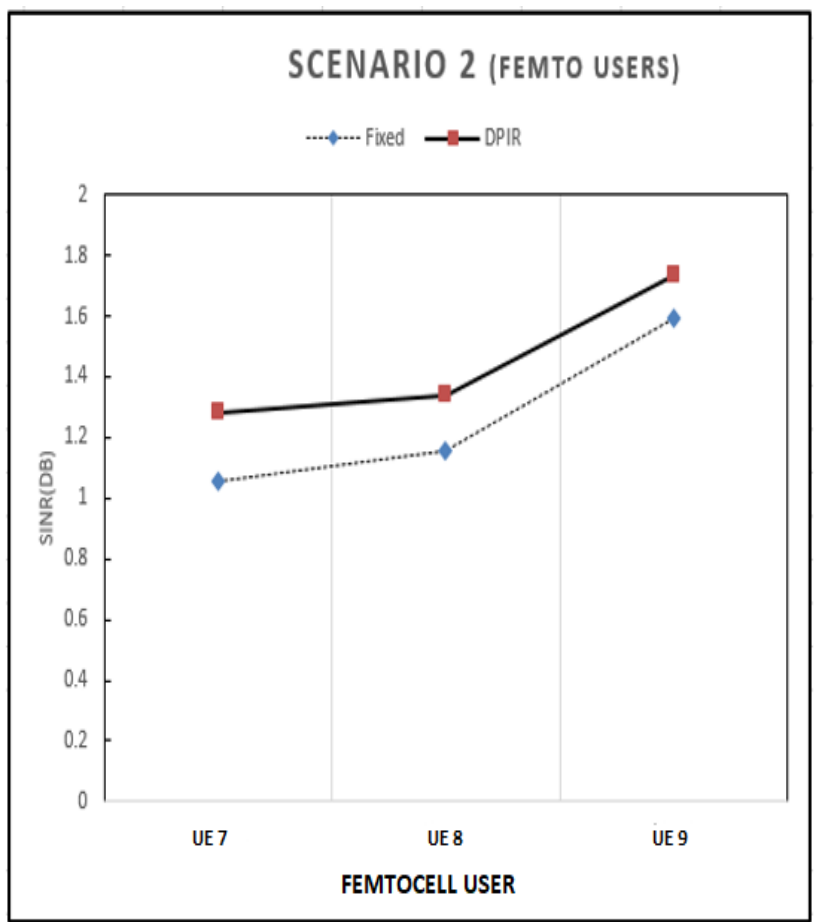

Figure 10: The effect of DAPIR on other users in macrocell based on Scenario 2

\subsection{Scenario 3}

In scenario 3, mixed traffic in which both MUE and FUEs are present in the conflicting zone between macrocell and femtocell. After applying DAPIR algorithm SINR value of traffic in the conflicting zone is improved but at the expense of increasing interference in macrocell.

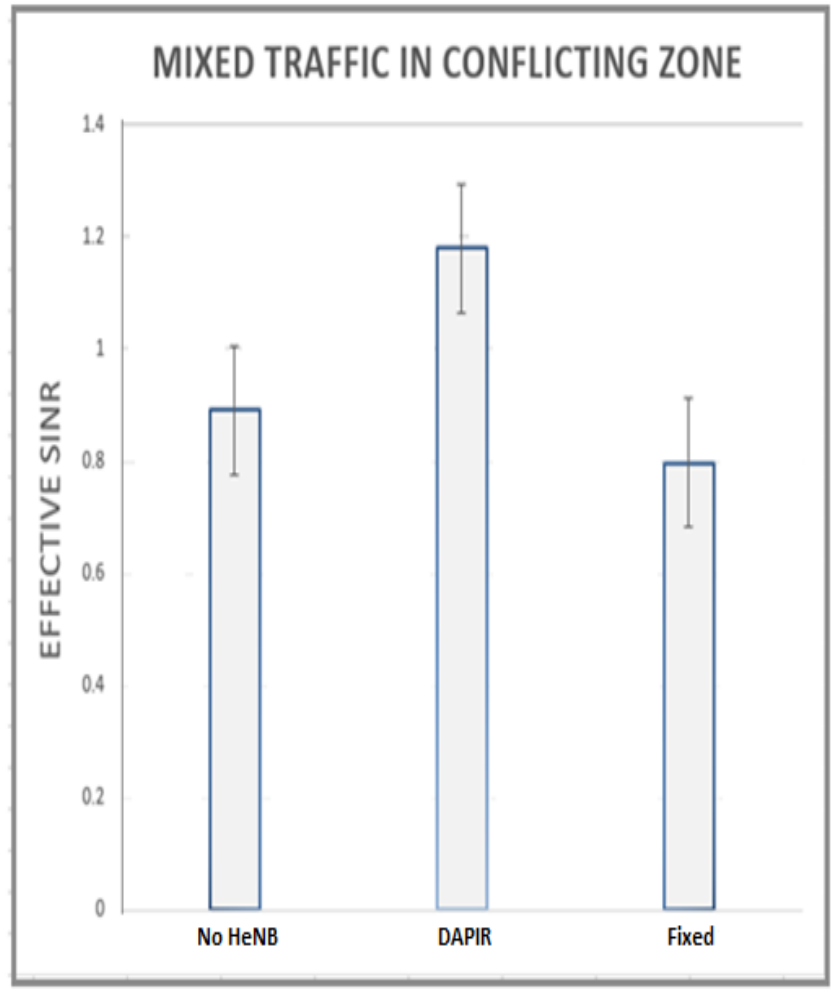

\section{Figure 11: SINR Results during Mixed Traffic in Conflicting Zone}

The results of scenario 3 clearly indicate that our scheme performs better than the other two. In overall results, DAPIR algorithms perform better and successfully decrease the interference level by increasing SINR values.

\subsection{CONCLUSION}

The performance of this scheme is analyzed and tested during considering various scenarios where possibilities of interference might occur. This scheme is compared with fixed power and no HeNB. The performance of our scheme is far better than the other two in most of the scenarios. The implementation issues of our control scheme and estimation of our two key decision-making parameters are discussed in detail. The CQI report generation capability is integrated into the system without additional costs.

\subsection{FUTURE WORKS}

The scheme is purely based on "Open Access Mode". Any users come in the coverage of femtocell enjoy its full resources. The idea behind this just increases coverage and capacity for the operator's point of view. This scheme is not very feasible for the private use of femtocell. Unauthorized users will also enjoy full resources. The further goal and target of our research are to extend the capabilities our proposed scheme considering "Hybrid Access Mode" by providing coverage to all users but preference must be given to the registered users only. Furthermore, the negative impact of an extended number of handover and increase the power of FBS on macrocell must be analyzed. Implement this scheme on real-time mobility model on 5G.

\subsection{ACKNOWLEDGEMENT}

Special thanks and appreciations for all those who contributed directly or indirectly in this research work. 


\section{REFERENCES}

[1] Ericsson official site, "Mobility Report," 2018. [Online]. Available:

https://www.ericsson.com/assets/local/mobilityreport/documents/2018/ericsson-mobility-reportnovember-2018.pdf. [Accessed: 22-Apr-2019].

[2] Ericsson official site, "The future of mobile subscriptions," 2018. [Online]. Available: https://www.ericsson.com/en/mobility-report/future-ofmobile-subscriptions. [Accessed: 23-Apr-2019].

[3] M. Al-Omari, A. R. Ramli, A. Sali, and R. S. Azmir, "Deployment of femtocell and its interference management approaches in LTE heterogeneous networks," J. Theor. Appl. Inf. Technol., vol. 87, no. 1, pp. 54-79, 2016.

[4] A. L. Yusof, S. S. Salihin, N. Ya'acob, and M. T. Ali, "Performance analysis of handover strategy in Femtocell network," J. Commun., vol. 8, no. 11, pp. 724-729, 2013.

[5] S. Palipana, Y. Zaki, U. Toseef, J. Chen, and C. Goerg, "Scalable and self-sustained algorithms for femto-cell interference mitigation," Lect. Notes Inst. Comput. Sci. Soc. Telecommun. Eng. LNICST, vol. 141, pp. 3-17, 2015.
[6] K. Senel and M. Akar, "A fair downlink power control algorithm for femtocell networks," IEEE Int. Conf. Control Autom. ICCA, vol. 2016-July, pp. 305-310, 2016.

[7] M. Susanto, D. Fauzia, Melvi, and S. Alam, "Downlink power control for interference management in femtocellmacrocell cellular communication network," in 2017 15th International Conference on Quality in Research (QiR) : International Symposium on Electrical and Computer Engineering, 2017, pp. 479-484.

[8] T. U. Hassan, F. Gao, B. Jalal, and S. Arif, "Interference management in femtocells by the adaptive network sensing power control technique," Futur. Internet, vol. 10, no. 4, 2018.

[9] D. Singhal, M. Kunapareddy, V. Chetlapalli, V. B. James, and N. Akhtar, "LTE-advanced: Handover interruption time analysis for IMT-A evaluation," 2011 Int. Conf. Signal Process. Commun. Comput. Netw. Technol. ICSCCN-2011, no. July, pp. 81-85, 2011.

[10] S. O. Aramide, B. Barakat, Y. Wang, S. Keates, and K. Arshad, "Generalized proportional fair (GPF) scheduler for LTE-A," 2017 9th Comput. Sci. Electron. Eng. Conf. CEEC 2017 - Proc., no. 1, pp. 128-132, 2017. 